\title{
An Approach to Improve Software Quality in Length Measurement Calibration
}

\author{
N. Greif, H. Schrepf, D. Richter \\ Physikalisch-Technische Bundesanstalt (PTB) \\ Abbestr. 2-12, 10587 Berlin, Germany; email: norbert.greif@ptb.de
}

\begin{abstract}
Software risks for incorrect gauge block calibrations in length measurement are identified. Technical recommendations are given to minimize these risks. Measures of integrating the recommendations into the practical work of calibration services are outlined.
\end{abstract}

\section{INTRODUCTION}

The setting-up of calibration services is a way to make high precision metrology accessible to industry. Calibration concentrates traditionally on the dissemination of the units of measurement at a high level of accuracy, using the advanced state of the art of metrology. Parts of measurement processes have gradually become supported by software or have been even replaced by it. Without reducing the accuracy requirements, another kind of problems has come up in metrology in general and calibration in particular: errors of measurement caused by software failures. As a consequence, this type of problems has to be dealt with in addition to the problems of precise measurements encountered anyway.

To verify or measure software quality, a system of clear, testable software requirements has to be established. However, requirements for measurement systems are usually available for the system as a whole but not explicitly for its software part. The metrologist cannot, therefore, specify software requirements in the form the software engineer needs to apply appropriate methods. This gap is of a basic nature.

Parallel gauge blocks are an important material measure in length measurement. They represent a certain length by the distance between two parallel boundary planes. The difference measurement (comparison between a gauge block under test and a reference standard) is performed repeatedly in the center and near the four corners. The requirements for the measurement conditions are very high, in particular concerning the temperature, the standard deviation of the length differences and the care with which the blocks must be handled.

\section{ADDITIONAL RISKS CAUSED BY SOFTWARE}

Concerning additional software risks, a distinction between the following five groups of problems seems to be useful: completeness, correctness and functionality of software; correctness of the code; data handling; proper use of software; and management questions.

Some details concerning the first group are outlined in the following. All the problems dealing with the adequate and correct coverage of expected functions by the software belong 
to this group. In particular, questions arising from the application of mathematical algorithms, including the mathematical background and numerical aspects, play an essential role. However, in gauge block calibration no mathematical problems are actually encountered. The functions supported by software are the control of gauge block comparisons and some aspects of measurement data processing. The controlling part has to ensure that the calibration process is performed in accordance with the prescribed steps. These steps can be altered depending on the calibration task and on intermediate measurement results. Alterations are

- surface positions used for the difference measurement;

- single or repeat measurements, conditions for repetitions;

- application or not of flattening correction;

- application or not of temperature correction.

Another sensitive aspect of the controlling software is the reliability under extreme conditions. This point is evidently a general one. Here we have the two points:

- correct execution of measurements, also under extreme but permissible conditions;

- rejection of execution or a warning in the case of inadmissible conditions or input data.

With respect to completeness of the software functions, risks exist when the following functions are not supported sufficiently: input, use and archiving of data sets of reference standards; input of parameters for the gauge block under test; control of the execution of measurements; print of test protocols, results and calibration certificates; and archiving of measurement results including a list of content.

\section{TECHNICAL RECOMMENDATIONS FOR DEVELOPING CALIBRATION SOFTWARE}

\section{Program correctness and reliability}

Software defects can be avoided to a large extent, when an application-dependent combination of the following elements is used in the software development process:

- a well-defined software development model (e.g. the life-cycle model of ISO/IEC 12207);

- a quality management system according to ISO 9001, ISO 9000-3;

- well-defined development methods (e.g. structured analysis and design);

- validated software tools if necessary.

Techniques of software product evaluation and testing are supported by several international standards (e.g. ISO/IEC 14598, ISO/IEC 9126, ISO/IEC 12119). An appropriate combination of the following techniques should preferably be applied to verify calibration software:

- human testing (design and code inspections, code walkthroughs, reviews);

- tool-supported static analysis of the nonexecutable software entity;

- tool-supported dynamic testing (black-box testing based on program specification, whitebox testing based on the internal structure of the program).

\section{Program identification and documentation}

To support the handling of different calibration programs and different versions of a program in calibration laboratories (for example, to ensure traceability of calibration results), each of the programs must be unambiguously identifiable. A minimal requirement is the indication of the program name, the version number and the manufacturer of the software.

The documentation is an essential part of each software product. In calibration laboratories, a documentation of high quality must be available for each calibration program. 


\section{User interface}

For calibration software the use of graphical user interfaces (GUIs) is recommended. The windowing system should follow approved standards. The application of acoustic and/or optical signals is recommended for certain dangerous situations (e.g. in the case of high variation of measurement values or inadmissible user inputs). At critical decision points in the user guidance process (e.g. change of standard probe data), the user should be requested to confirm the transactions. Terms have to be used in a consistent and unambigous manner.

\section{Data handling}

Recommendations for data handling comprise the domains of data input, transfer, storage, and security. We emphasize that plausibility tests should be required for each data input. Above all the compliance of inputs with boundary values or intervals of parameters and the unambiguousness of identification numbers (for example of standard probes) must be tested.

Critical data (e.g. deviations of standard probes) must be protected against intentional and unintentional manipulations. Measures to secure data are password protection, specific rights for accessing different sets of standard probes, and encoded storage on hard disk. Changes of critical data have to be confirmed before they are transferred to the data archive.

\section{Measurement data processing and presentation of results}

Data processing must be organized in a way that ensures the traceability of calibration results. The following recommendations in particular should be considered:

- Processing of the measurement values only in the case of a correct measurement.

- Not only the final (computed) measurement results should be displayed and archived but also the original raw data of the measurement.

- Each of the measurement values should be tested for plausibility before it is processed.

- In the case of large deviations of measurement values, a warning should be displayed.

- Rejection of measurements if a set of standard probes is used which has no valid calibration.

- In addition to the automatic transfer of measurement data, the program should offer a mode for manual input of raw data.

The record of the program run should contain all important data (e.g. date, testing engineer, parameters of the measuring instrument, temperature, standard probe data, data of the gauge block under test). Each of the measurement values has to be assigned unambiguously to a specific measurement position on the gauge block surface. The program should give an overview of all sets of standard probes if necessary. When the measurement results are stored, subsequent printing of calibration certificates should be possible at any time.

\section{PRACTICAL APPROACHES OF SOFTWARE QUALITY ASSURANCE}

After risks have been identified and technical measures recommended, the next step is to propose practically applicable approaches to integrate the recommendations into the practical work of calibration services. We propose two different approaches complementing one another.

\section{Checklist}

With respect to gauge block calibration, it has turned out that working with a checklist representing a set of minimum mandatory requirements for the software is an 
appropriate means to enhance the reliability of software. A checklist can be used both when a calibration service is certified by external experts and when a self-check is made.

Prerequisite for the work with a checklist is that an appropriate list of requirements is drawn up and that rules are formulated how to prove compliance with the requirements.

The elaboration of a set of minimum mandatory requirements has been based on the risk analysis and on the technical recommendations. It has so far ended in a list of 37 requirements arranged in 7 different groups (identification, documentation, functions to fulfil, usability, data handling, security, transparency and repeatability). For illustration purposes, we list five requirements of the group 'usability':

1. Notions of the user interface as well as of the documentation that are important for the measurement are to be applied correctly, uniquely and consistently.

2. The software must not break down even in the case of nonsensical operation.

3. Each change of data has to be either stored permanently or rejected immediately. The software must not process data feed in incompletely.

4. The permanent change of sensitive data (e.g. reference parameter) has to be confirmed.

5. An error list including recommendations to continue the work must be available.

To determine whether or not a program complies with the requirements, concrete instructions are necessary. For practical reasons, the instructions must be such that a nonspecialist of software is able to understand and apply them.

\section{Standardized testing technology and test case design}

To guarantee the correctness and reliability of calibration software in a uniform way, the application of a standardized testing technology and the construction of standardized test cases are required. A standardized interpretation of test results would ensure the comparability of test results among all calibration services. When standardized test cases are run successfully, a uniform minimal level of software correctness is achieved.

There are two basic ways of constructing test cases for testing the correctness of calibration software. The first consists in designing test cases systematically according to the well-studied methods of software testing. Another way to construct test cases is based on the experience gained by the calibration services from typical errors.

A preparatory task to establish a standardized testing technology is the determination of relevant testing areas in the calibration process. Examples of such areas are the measurement evaluation, the data management process, protection against manipulation, and controlling of different kinds of calibration. To test for example the processing of measured length differences, test cases have to be constructed considering the different parameters of the computation algorithm. These parameters include

- kind of measurement: single measurement or multiple sensor touching with averaging;

- measurement position: five positions at gauge block (computation of the total deviation range) or only center position (computation of the deviation range for the center position);

- computation with flattening correction or not;

- computation with temperature correction or not.

Because of the independence of the parameters, a subset of four classes of test cases seems to be sufficient to test the correctness of the computation algorithm.

\section{Acknowledgement}

This study was stimulated by the German Calibration Service (DKD), Technical Committee for Length Measurement. The authors are much obliged for support and advice of the PTB Laboratory for Interferential Length Measurement headed by Dr. Gerhard Bönsch. 